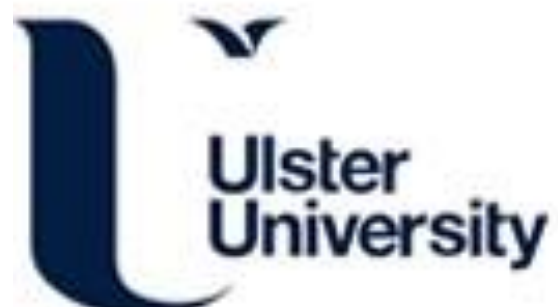

\section{Shoulder and hip roll differences between breathing and non-breathing conditions in front crawl swimming}

Psycharakis, S., \& McCabe, C. (2011). Shoulder and hip roll differences between breathing and non-breathing conditions in front crawl swimming. Journal of Biomechanics, 44(9), 1752-1756.

https://doi.org/10.1016/j.jbiomech.2011.04.004

Link to publication record in Ulster University Research Portal

\section{Published in:}

Journal of Biomechanics

Publication Status:

Published (in print/issue): 03/06/2011

DOI:

10.1016/j.jbiomech.2011.04.004

\section{Document Version}

Publisher's PDF, also known as Version of record

\section{General rights}

Copyright for the publications made accessible via Ulster University's Research Portal is retained by the author(s) and / or other copyright owners and it is a condition of accessing these publications that users recognise and abide by the legal requirements associated with these rights.

\section{Take down policy}

The Research Portal is Ulster University's institutional repository that provides access to Ulster's research outputs. Every effort has been made to ensure that content in the Research Portal does not infringe any person's rights, or applicable UK laws. If you discover content in the Research Portal that you believe breaches copyright or violates any law, please contact pure-support@ulster.ac.uk. 


\title{
Shoulder and hip roll differences between breathing and non-breathing conditions in front crawl swimming
}

\author{
Stelios G. Psycharakis ${ }^{\mathrm{a}, \mathrm{b}, *}$, Carla McCabe ${ }^{\mathrm{a}, \mathrm{b}}$ \\ ${ }^{a}$ School of Life, Sport and Social Sciences, Edinburgh Napier University, Sighthill Campus, Sighthill Court, Edinburgh, EH11 4BN, Scotland, UK \\ ${ }^{\mathrm{b}}$ Centre for Aquatics Research and Education, University of Edinburgh, Edinburgh, UK
}

\section{A R T I C L E I N F O}

\section{Article history:}

Accepted 4 April 2011

\section{Keywords:}

Biomechanics

Kinematics

Body roll

Breath-holding

Swimming speed

\begin{abstract}
A B S T R A C T
The effects of breathing on body roll have been previously investigated for the roll of the whole trunk only. The purposes of this study were: to calculate separately the shoulder roll (SR) and hip roll (HR) of swimmers during front crawl for non-breathing and preferred-side breathing conditions; to assess the differences in the magnitude and temporal characteristics of these variables between non-breathing and preferred-side breathing conditions; and to examine their association with swimming performance (indicated by swimming speed). Twelve male swimmers who competed at national and international level performed two maximum $25 \mathrm{~m}$ front crawl trials: one non-breathing and one with breathing to their preferred side. Performance was recorded with four below and two above water synchronised cameras. SR and HR in both trials were calculated for the breathing and non-breathing sides. The timings of SR and HR peaks to each side and at the positions of neutral roll were also calculated. Swimming speed was significantly slower in the breathing trial $(p<0.01)$. Swimmers rolled their shoulders and hips to the breathing side significantly more in the breathing than in the non-breathing trial (SR: $p<0.01$; HR: $p=0.03$ ). Nevertheless, there were no significant differences in the overall SR or HR between these trials. In the breathing trial, SR was higher in the breathing than in the non-breathing side $(p<0.01)$ but HR was not significantly different $(p=0.07)$. There was no evidence to suggest that temporal characteristics of SR or HR were associated with swimming performance.
\end{abstract}

(c) 2011 Elsevier Ltd. All rights reserved.

\section{Introduction}

The alternation between left and right arm strokes in front crawl swimming is accompanied by rotations of the trunk around its longitudinal axis. These rotations are commonly known as body roll (BR). BR has important functions in front crawl swimming, such as facilitating the breathing action (Yanai, 2001) and the recovery of the arm (Counsilman, 1968), as well as affecting the underwater hand path, therefore contributing to hand velocity (Payton et al., 2002). It has been suggested that BR is linked to swimming performance; Psycharakis and Sanders (2008) indicated that faster swimmers rolled their shoulders less than slower swimmers during a $200 \mathrm{~m}$ front crawl test, while Yanai (2003) reported that swimmers rolled their shoulders $9^{\circ}$ less when they increased speed from 1.3 to $1.6 \mathrm{~m} \mathrm{~s}^{-1}$. Other authors have stated that BR might assist in increasing propulsion or decreasing drag forces (Cappaert et al., 1995; Castro et al., 2003),

\footnotetext{
* Corresponding author at: School of Life, Sport and Social Sciences, Edinburgh Napier University, Sighthill Campus, Sighthill Court, Edinburgh, EH11 4BN, Scotland, UK. Tel.: + 441314552215 ; fax: +441314552291.

E-mail address: S.Psycharakis@napier.ac.uk (S.G. Psycharakis).
}

and in reducing the risk of developing shoulder injuries (Weldon and Richardson, 2001).

The breathing actions in front crawl swimming might cause alterations on stroke mechanics, for example an increase in hydrodynamic drag and BR (Pendergast et al., 1977), and a decrease in horizontal velocity (Castro et al., 2006; Payton et al., 1999). Despite the potential links between BR, breathing actions and front crawl swimming performance, this area has been the topic of only a few studies (Beekman and Hay, 1988; Castro et al., 2006; Payton et al., 1999). In the latter studies, researchers calculated BR for the whole trunk, based on the assumption that the trunk rotates longitudinally as a rigid segment. For the calculation of trunk roll, a wooden fin was attached on each swimmer's back and swimmers swam towards a poolside camera. Using this camera, trunk roll was calculated with two-dimensional methods as the angle between the fin and the vertical axis (Fig. 1).

The findings from the above studies were inconclusive. Greater trunk roll in the breathing trial was reported by Payton et al. (1999), Castro et al. (2006) for swimmers swimming at moderate and fast intensities and Beekman and Hay (1988) for swimmers with a shoulder injury. On the contrary, no differences in trunk roll between breathing and non-breathing trials were reported by Castro et al. (2006) for swimmers swimming at low intensities 


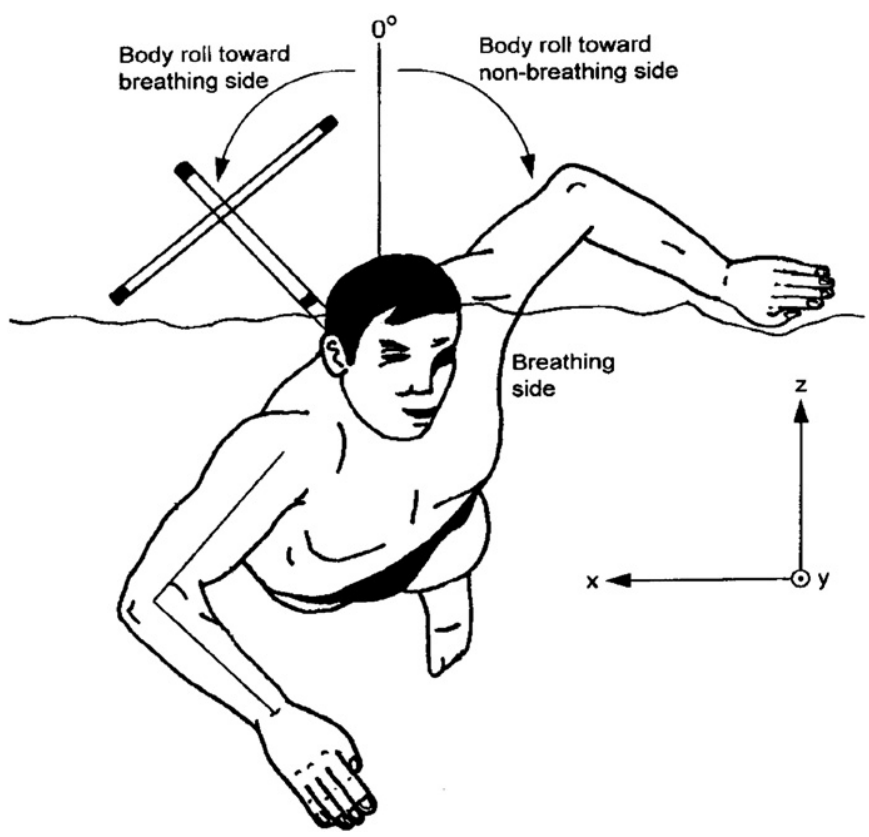

Fig. 1. Two-dimensional method used for the calculation of the roll of the trunk in previous studies. Trunk roll was defined as the angle between the fin and the vertical axis for the $X Z$ plane. (Adapted from Payton et al., 1999).

and for triathletes and Beekman and Hay (1988) for injuryfree swimmers. The discrepancies across studies could perhaps be attributed to factors such as less consistent roll patterns of injured swimmers and triathletes compared to healthy swimmers (Beekman and Hay, 1988), large range of swimming speeds tested (1.13-1.96 $\mathrm{m} \mathrm{s}^{-1}$ ) and high variability of speeds recorded for a given swimming intensity.

Although the above studies produced some noteworthy findings, the assumption that the trunk rotates as a unit in front crawl might not be tenable and therefore, the calculation of trunk roll only might mask differences in the magnitude of shoulder roll (SR) and hip roll (HR). Psycharakis and Sanders (2008) calculated separately SR and HR for non-breathing front crawl swimming, and indicated that shoulders rolled significantly more than the hips and that between swimmer differences existed in the temporal characteristics of SR and HR. Psycharakis and Sanders (2010) suggested that the effects of breathing actions should be calculated separately for SR and HR, to explore the roll differences between breathing and non-breathing stroke cycles (SCs) and their association with swimming performance.

The purposes of the present study were: to calculate SR and HR separately during maximum effort front crawl swimming for non-breathing and preferred-side breathing conditions; to assess the differences in the magnitude and temporal characteristics of these variables between non-breathing and preferred-side breathing conditions; and to examine their association with swimming performance (indicated by swimming speed).

\section{Methods}

\subsection{Participants}

Twelve male swimmers competing at national and international level participated in this study $(18.9 \pm 2.4$ years; $183.4 \pm 8.1 \mathrm{~cm} ; 73.5 \pm 10.2 \mathrm{~kg}$; personal best performance in $50 \mathrm{~m}$ front crawl: $25.3 \pm 0.9 \mathrm{~s}$ or $86.0 \pm 3.2 \%$ of the world record average speed). The swimmers were free from injuries and were specialised in front crawl events. The test procedures were approved by the institutional ethics committee and written informed consent forms were obtained before the study commenced.

\subsection{Protocol}

All tests were conducted in a $25 \mathrm{~m}$ indoor pool. Following a personalised warm-up, swimmers performed two maximum $25 \mathrm{~m}$ front crawl trials in randomised order; one trial with breathing to their preferred side and one nonbreathing trial. A push start was used to eliminate the influence of the dive on the kinematics of the SCs analysed. Six stationary, synchronised and genlocked JVC-KY32CCD cameras (four below and two above the water; frequency $50 \mathrm{~Hz}$ ) recorded the trials. The camera and calibration set-up is described in detail by Psycharakis and Sanders (2008) (also see Appendix 1.1). Psycharakis et al. (2005) indicated that this set-up has high accuracy and reliability, produces small and acceptable calculation errors and negligible image distortion and refraction (also see Appendix 1.2).

\subsection{Data processing}

Two SCs were analysed, one for the breathing and one for the non-breathing trial. These SCs were defined as the period between two consecutive hand entries of the same hand, at the side of preferred breathing. For example, for swimmers breathing to the left side, the SC would be defined from left hand entry to left hand re-entry, for both the breathing and non-breathing trials.

Nineteen body landmarks were digitised for each field (vertex; shoulders, elbows, wrists, hips, knees, ankles, metatarsophalangeal joints; end of middle fingers and big toes) using the Ariel Performance Analysis System (APAS, Ariel Dynamics Inc., California). The three-dimensional reconstruction was performed as described by Psycharakis and Sanders (2008) (also see Appendix 1.3).

\subsection{Data analysis}

Shoulder and hip roll were calculated as described by Psycharakis and Sanders (2008) (also see Appendix 1.4). In both breathing and non-breathing trials the total SR and HR were calculated, defined as the sum of the SR or HR, respectively, to the breathing and non-breathing sides. SR and HR were also calculated separately for the breathing and non-breathing sides. The SR and HR calculations were abbreviated using 'SR' or 'HR' and two letters, with the first letter indicating the side (breathing: $\mathrm{B}$; non-breathing: $\mathrm{N}$; total roll: $\mathrm{T}$ ) and the second letter the trial (breathing: $\mathrm{B}$; non-breathing: $\mathrm{N}$; see Appendix 1.5 for an example). With respect to temporal characteristics, the timings of the SR and HR peaks to each side were calculated as a percentage of the SC (\%SC), while the timings of the two neutral roll positions $\left(0^{\circ}\right)$ were also determined, as suggested by Psycharakis and Sanders (2008). The instantaneous swimming speed of the centre of mass $\left(\mathrm{m} \mathrm{s}^{-1}\right)$ was calculated with the use of the elliptical zone method and the procedures were described by Psycharakis et al. (2010). The average swimming speed for each trial was then calculated by taking the mean of instantaneous speed values for each SC. Repeated digitising of a SC indicated good reliability for all variables (Appendix 1.6).

\subsection{Statistical analysis}

The Shapiro-Wilk test indicated that the data were normally distributed. Paired samples $t$-tests were used to assess the significance of the differences between the following: the breathing and the non-breathing trial for each variable; the SR values on the breathing and the non-breathing sides for each trial; and the HR values on the breathing and the non-breathing sides for each trial; the SR and HR values for each side and for each trial. The above tests were performed for both the roll magnitude and timing (when applicable). To provide a further indication of the magnitude of the differences, the effect sizes (d) for all the statistically significant differences were calculated based on Cohen's suggestions (1988), with each pooled SD being calculated as described by Field (2005). In line with Cohen's recommendations, effect sizes of a magnitude of $0.2,0.5$ and 0.8 were considered small, moderate and large, respectively. Pearson's product moment correlation coefficient $(r)$ was calculated to assess the nature and strength of correlations between SR/HR and swimming speed for each side and for each trial. For all statistical calculations, significance was accepted at $p<0.05$. Statistical analysis was conducted with the Statistical Package for Social Sciences software (version 14.0, SPSS Inc., Chicago).

\section{Results}

\subsection{Differences between breathing and non-breathing trials}

The mean swimming speed was significantly higher $\left(t_{(11)}=\right.$ $-3.51, p<0.01)$ in the non-breathing $\left(1.81 \pm 0.08 \mathrm{~m} \mathrm{~s}^{-1}\right)$ than in the breathing trial $\left(1.76 \pm 0.07 \mathrm{~m} \mathrm{~s}^{-1}\right)$. There was a moderate effect size $(d=0.64)$ and the mean difference was $0.05 \pm 0.05 \mathrm{~m} \mathrm{~s}^{-1}$ 
( $2.8 \pm 2.8 \%$ of the breathing trial speed). Figs. 2 and 3 show the magnitude of SR and $\mathrm{HR}$, respectively. SR-BB was significantly higher than SR-BN $\left(t_{(11)}=4.12, p<0.01\right)$. The effect size was large $(d=1.04)$ and the mean difference was $8.0 \pm 6.7^{\circ}(15.4 \pm 12.9 \%$ of the SR-BN value). HR-BB was significantly higher than HR-BN $\left(t_{(11)}=2.51, p=0.03\right)$. There was a moderate effect size $(d=0.58)$ with the mean difference being $4.2 \pm 5.7^{\circ}(20.6 \pm 27.9 \%$ of the HR-BN value). No other significant differences were found in the magnitude of SR or HR between the two trials.

Table 1 shows the values and paired samples $t$-tests between breathing and non-breathing trials for the temporal characteristics of SR and HR. Swimmers reached the peak SR-BB significantly earlier than the peak SR-BN $\left(t_{(11)}=-3.07, p=0.01\right)$. The effect size

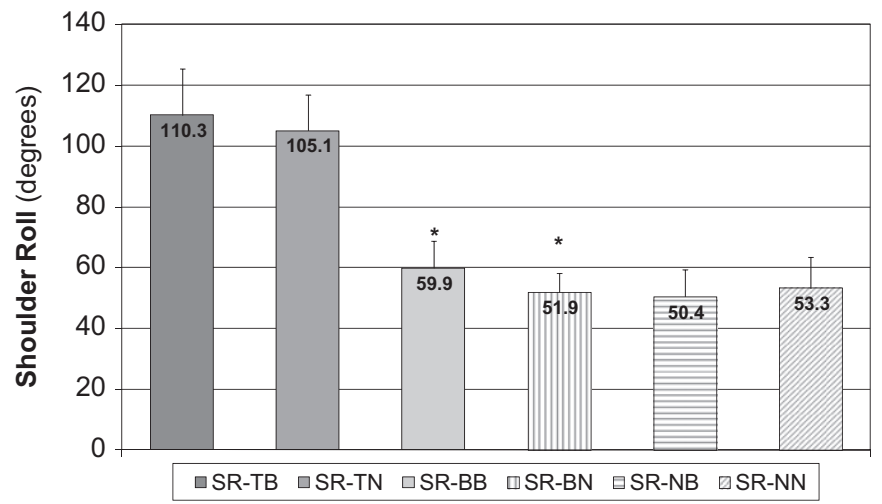

Fig. 2. Magnitude of shoulder roll for breathing and non-breathing sides and trials. For explanation of abbreviations see Section 2.3. Note: “*” indicates that the two variables are significantly different.

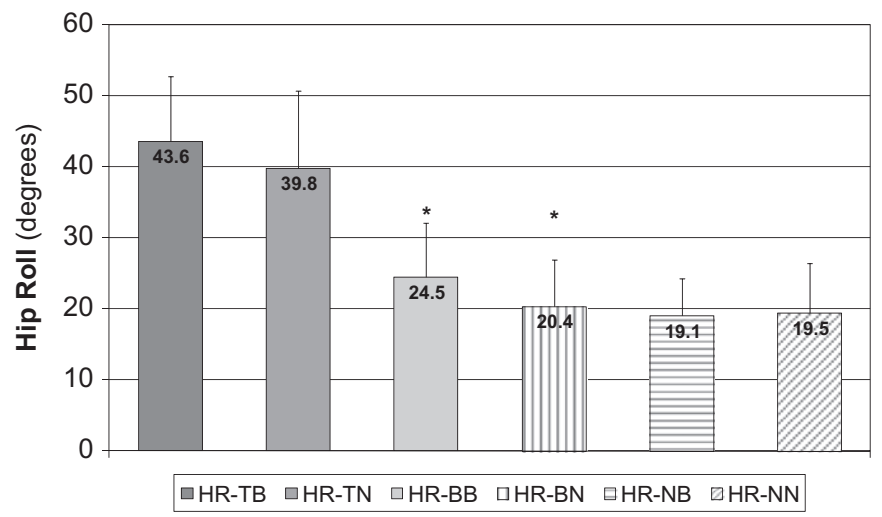

Fig. 3. Magnitude of hip roll for breathing and non-breathing sides and trials. For explanation of abbreviations see Section 2.3. Note: “*” indicates that the two variables are significantly different. was large $(d=0.99)$ and the mean difference was $5.9 \pm 6.7 \% \mathrm{SC}$ ( $7.1 \pm 8.0 \%$ of the SR-BN value).

\subsection{Differences between and within breathing and non-breathing sides for each trial}

Table 2 shows the results for the paired samples $t$-tests comparing the roll values between the breathing and non-breathing sides for each trial. SR was consistently higher than HR in each trial for both sides as well as the total values $\left(10.82 \leq t_{(11)} \leq\right.$ 28.82, $p<0.01)$. The effect sizes of the differences were large $(4.26 \leq d \leq 5.74)$ and the magnitude of SR was more than double that of HR. For the breathing trial, SR-BB was significantly higher than SR-NB $\left(t_{(11)}=3.48, p<0.01\right)$ with a large effect size $(d=1.06)$ and the mean difference being $9.5 \pm 9.4^{\circ}(18.8 \pm 18.7 \%$ of the SR-NB value). No significant differences were found between the timings of SR and HR at the positions of neutral roll.

\subsection{Correlations between roll and swimming performance}

Swimming speed had a positive significant correlation with SR-NB $(r=0.75, p<0.01)$ and with SR-NN $(r=0.60, p=0.04)$. No other significant correlations were found between swimming speed and the magnitude of SR or HR (for either side or the total roll). between swimming speed and the timing of SR or HR (for the positions of peak or neutral roll).

\section{Table 2}

Significant levels obtained through paired samples $t$-tests for the following comparisons: between the SR values on the breathing and the non-breathing sides for each trial; and between the HR values on the breathing and the nonbreathing sides for each trial; between the SR and HR values for each side and for each trial. For explanation of abbreviations see Section 2.3 .

\begin{tabular}{lcl}
\hline Roll variables compared & $\boldsymbol{P}$ value for magnitude & $\boldsymbol{P}$ value for timing \\
\hline Breathing trial & & \\
SR-BB with SR-NB & $<0.01^{*}$ & Not applicable \\
HR-BB with HR-NB & 0.07 & Not applicable \\
SR-TB with HR-TB & $<0.01^{*}$ & Not applicable \\
SR-BB with HR-BB & $<0.01^{*}$ & 0.65 \\
SR-NB with HR-NB & $<0.01^{*}$ & 0.51 \\
Non-breathing trial & & \\
SR-BN with SR-NN & 0.69 & Not applicable \\
HR-BN with HR-NN & 0.70 & Not applicable \\
SR-TN with HR-TN & $<0.01^{*}$ & Not applicable \\
SR-BN with HR-BN & $<0.01^{*}$ & 0.15 \\
SR-NN with HR-NN & $<0.01^{*}$ & 0.84 \\
\hline
\end{tabular}

* Significant at $p<0.05$.

Table 1

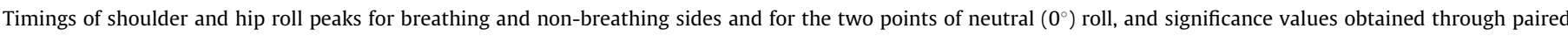
samples $t$-tests for the comparison of roll variables between breathing and non-breathing trials. For explanation of abbreviations see Section 2.3 .

\begin{tabular}{|c|c|c|c|c|}
\hline Breathing trial & $\begin{array}{l}\text { Timing of peak roll } \\
\text { (\% of SC) }\end{array}$ & $\begin{array}{l}\text { Non-breathing } \\
\text { trial }\end{array}$ & $\begin{array}{l}\text { Timing of peak roll } \\
\text { (\% of SC) }\end{array}$ & $\begin{array}{l}\text { Paired sample } \\
t \text {-tests ( } p \text { values) }\end{array}$ \\
\hline SR-NB & $28.7 \pm 9.4$ & SR-NN & $31.4 \pm 7.2$ & 0.18 \\
\hline SR-BB & $78.3 \pm 7.3$ & SR-BN & $84.2 \pm 4.3$ & $0.01^{*}$ \\
\hline SR-Neutral 1 & $7.0 \pm 6.2$ & SR-Neutral 1 & $6.2 \pm 4.9$ & 0.41 \\
\hline SR-Neutral 2 & $53.5 \pm 4.7$ & SR-Neutral 2 & $55.2 \pm 4.8$ & 0.09 \\
\hline HR-NB & $29.9 \pm 10.8$ & HR-NN & $30.9 \pm 10.3$ & 0.52 \\
\hline HR-BB & $79.7 \pm 9.6$ & HR-BN & $71.3 \pm 8.7$ & 0.28 \\
\hline HR-Neutral 1 & $6.4 \pm 7.1$ & HR-Neutral 1 & $5.9 \pm 5.4$ & 0.70 \\
\hline HR-Neutral 2 & $49.8 \pm 7.2$ & HR-Neutral 2 & $52.5 \pm 7.7$ & 0.26 \\
\hline
\end{tabular}

* Significant at $p<0.05$ 


\section{Discussion}

The aim of this study was to assess the differences in the magnitude and temporal characteristics of SR and HR between non-breathing and preferred-side breathing trials, and to explore any associations with swimming performance. Some notable significant differences with large effect sizes were found between SR and HR, as well as for the magnitude and timing of roll between breathing and non-breathing trials and sides.

\subsection{Differences between breathing and non-breathing trials}

The total amount of SR and HR was not different between breathing and non-breathing trials, despite a tendency for higher values in the breathing trial. Nevertheless, there were some noteworthy changes in stroke mechanics. Swimmers rolled their shoulders/hips to the breathing side significantly more in the breathing than in the non-breathing trial, most probably to facilitate the turning of the head for the breathing action as suggested by Yanai (2001) and Payton et al. (1999). This notion is also supported by experimental data in butterfly swimming, where Barbosa et al. (1999) reported that swimmers tended to roll their bodies around their longitudinal axis when using lateral inspiration, with no such pattern observed for frontal inspiration. Although it seems logical that the swimmers in the present study rolled more to the breathing side in the breathing trial to facilitate the head turning action, it could perhaps be considered a little surprising that this increase did not lead to an increase in the total SR or HR in the breathing trial. Closer inspection of the data showed that swimmers tend to roll to the non-breathing side less in the breathing than in the non-breathing trial. Although the latter differences were not statistically significant, this trend might have contributed to the total roll not being significantly different between the two trials. One should also not rule out the possibility that, given the tendency for higher total roll values in the breathing trial, significant differences could be found in the total roll between breathing and non-breathing trials if a larger number of swimmers were tested.

Swimming speed decreased significantly in the breathing trial. This is in agreement with the findings of Castro et al. (2006), but not those of Payton et al. (1999). However, one of the selection criteria in the latter study was that the SCs to be analysed would be less than $1 \%$ different in swimming speed, and this is the reason that subsequently no significant differences in speed were found. The data of the present study do not seem to provide strong indications regarding how body roll might have affected swimming speed. One possible explanation could be related to the trunk 'twist' values, which represent the differences between the magnitude of SR and HR. Assuming that swimmers reach the peak SR at each side at the same time as the peak HR, an increased trunk twist would imply a larger frontal surface area and, therefore, increased active drag and resistive forces for the positions of peak roll (Cappaert et al., 1995). In line with this, Yanai (2003) suggested that competitive swimmers might be using the trunktwist motion effectively, to prevent the amount of fluid forces wasted in non-propulsive directions from increasing. In the present study there was a marginal increase in trunk twist during the breathing trial for the trunk twist to the breathing side $\left(3.9^{\circ}\right)$ and the overall trunk twist $\left(1.3^{\circ}\right)$, but not for the trunk twist to the non-breathing side $\left(-2.5^{\circ}\right)$. Although these values could suggest a slight increase in resistive forces for some positions, they are quite small and close to the digitising reliability errors and, thus, any interpretations should be rather conservative. Moreover, although the assumption that the swimmers reach peak SR and HR at the same time could be accepted for the present study (given that there were no significant differences in the timings of SR and HR on each side, as shown in Table 2), it must be noted that this assumption might ignore individual differences. To improve the understanding of the influence of BR changes between breathing and non-breathing conditions on swimming speed it is suggested that more variables that could be related to BR are considered in futures studies, such as the propulsive and drag forces, the displacement and speed of the arms and hands, the angular or linear roll velocity and the kinematic characteristics of the lower extremities.

Swimmers reached the peak SR to the breathing side earlier when breathing than when not breathing. While this could be related to the duration of the SC, a paired samples t-test showed no significant differences in SC time between breathing and nonbreathing trials. For the SCS analysed in the present study, swimmers rolled first to the non-breathing side and then to the breathing side. Moreover, they showed a tendency for lower SR magnitude and earlier SR peak to the non-breathing side in the breathing than the non-breathing trial. It could be argued that this tendency might have contributed to the earlier timing of the SR peak in the breathing trial. The turning action of the head when taking a breath is expected to affect the roll of the shoulders and, therefore, this might also have contributed to the earlier SR peak in the breathing trial. Given that, as discussed below, there was no correlation between speed and the temporal characteristics of roll, it is not clear if the earlier SR peak in the breathing trial affected performance. This warrants further investigation with consideration of other factors that might influence the SR/HR temporal characteristics, such as the head movement and variables that are related to differences in motor coordination and roll rhythms.

\subsection{Differences between and within breathing and non-breathing sides for each trial}

In both trials swimmers rolled their shoulders more than their hips, confirming that SR and HR must be examined separately to assess accurately their influence on swimming performance. This is in agreement with Psycharakis and Sanders (2008), who found swimmers to roll their shoulders approximately twice as much as the hips, with the total SR ranging from $106.1 \pm 7.8^{\circ}$ to $107.4 \pm 7.9^{\circ}$. Although these authors reported larger HR values when the speed dropped during a $200 \mathrm{~m}$ swim, their values for the first $50 \mathrm{~m}$ (HR: $44.5 \pm 12.5^{\circ}$, speed: $1.68 \pm 0.05 \mathrm{~m} \mathrm{~s}^{-1}$ ) were similar to the HR values in the present study.

In the breathing trial, swimmers rolled their shoulders significantly more to the breathing than the non-breathing side. For the same trial, despite approaching significance, HR was not significantly different between the breathing and non-breathing sides. This suggests that the technique used to perform the breathing action appears to affect more the SR than the HR in terms of symmetry between the breathing and non-breathing sides. It should be noted that roll symmetry between breathing and non-breathing sides might also be affected by factors other than the breathing actions, such as swimmers' handedness and lateral dominance (Psycharakis and Sanders, 2008).

\subsection{Correlations between roll and swimming performance}

Swimming speed was not correlated with the magnitude of total SR or HR. The former is not in agreement with the findings of Psycharakis and Sanders (2008), who reported that in three of the four $50 \mathrm{~m}$ lengths of a $200 \mathrm{~m}$ swim, speed had a negative significant correlation with SR. A possible explanation for this discrepancy could be the difference in the events and swimming speeds in the two studies, with speeds in the latter study ranging from $1.68 \pm 0.05 \mathrm{~m} \mathrm{~s}^{-1}$ in the first $50 \mathrm{~m}$ to $1.45 \pm 0.06 \mathrm{~m} \mathrm{~s}^{-1}$ in the last $50 \mathrm{~m}$. Considering that SC times are longer for slower 
speeds, it could be assumed that slower swimmers tend to roll their shoulders more when more time is spent in a SC when swimming at sub-maximal speeds. Although this needs to be explored in depth in future studies, it seems that for maximal speeds the amount of total SR is not a factor directly associated with swimming performance. It should also be pointed out that breathing frequency is normally lower in short than long distance events, which could also be a factor affecting the differences in stroke kinematics between these events. In line with Psycharakis and Sanders (2008) there were no significant differences in the temporal characteristics of SR and HR and no correlations with swimming speed, providing no evidence that the shoulders leading or trailing the hips might be advantageous for swimming performance.

Swimming speed had a positive correlation with the magnitude of SR at the non-breathing side in both trials. For the breathing trial, given that the SR in the non-breathing side was less than in the breathing side, the former correlation suggested that faster swimmers might have had lower differences than slower swimmers in side-to-side SR. To test this possibility, swimming speed was correlated with the absolute values of the differences in SR between the breathing and the non-breathing sides. This correlation was not significant, providing no evidence that SR symmetry between the breathing and non-breathing sides is associated with performance. Future studies could improve the understanding for the reasons for any correlations between swimming speed and SR at the non-breathing side, by analysing the changes in roll and their association with changes in threedimensional intra-cycle velocity and acceleration for different phases of the SC, with particular interest in the positions of peak roll.

Due to space constraints of the swimming pool and the threedimensional analysis set-up, one SC was analysed for each maximum trial in the present study. Considering the level of the swimmers tested and based on evidence from previous BR studies, it would not be unreasonable to assume that these swimmers had established consistent roll patterns for swimming at maximum speed. Nevertheless, for the purposes of confirming and generalising the existing findings, researchers could analyse more data for swimmers swimming at both sprint and distance paces. Moreover, given that male swimmers have been tested in the majority of the studies in this area, roll data on female swimmers is warranted. The participants in the present study had primarily unilateral breathing patterns. It would be interesting to assess any differences in roll kinematics between unilateral and bilateral breathing swimmers for breathing and non-breathing conditions, and their links with swimming performance.

\section{Conclusion}

Swimming speed was higher in the non-breathing than in the breathing trial. Swimmers rolled more to the breathing side when breathing, but with no significant differences in the overall SR or HR. In the breathing trial, SR was higher in the breathing than in the non-breathing side but HR was not significantly different between the two sides. SR to the breathing side occurred earlier in the breathing than the non-breathing SC. There was no evidence to suggest that temporal characteristics of SR or HR were associated with swimming performance.

\section{Conflict of interest statement}

The authors do not have any conflict of interest.

\section{Acknowledgments}

The authors would like to thank Prof. Ross Sanders and Georgios Machtsiras for their contribution in the data processing of this study.

\section{Appendix A. Supplementary Materials}

Supplementary data associated with this article can be found in the online version at doi:10.1016/j.jbiomech.2011.04.004.

\section{References}

Barbosa, T., Sousa, F., Vilas-Boas, J.P., 1999. Kinematic modifications induced by the introduction of the lateral inspiration in butterfly stroke. In: Keskinen, K.L. Komi, P.V., Hollander, A.P. (Eds.), Biomechanics and Medicine in Swimming VIII. University of Jyvaskyla Publications, Jyvaskyla, pp. 15-19.

Beekman, K.M., Hay, J.G., 1988. Characteristics of the front crawl techniques of swimmers with shoulder impingement syndrome. Journal of Swimming Research 4 (3), 15-21.

Cappaert, J.M., Pease, D.L., Troup, J.P., 1995. Three-dimensional analysis of the men's 100-m freestyle during the 1992 Olympic games. Journal of Applied Biomechanics 11, 103-112.

Castro, F., Minghelli, F., Floss, J., Guimaraes, A., 2003. Body roll angles in front craw swimming at different velocities. In: Chatard, J.C. (Ed.), Biomechanics and Medicine in Swimming IX. University of St. Etienne Publications, St. Etienne pp. 111-114.

Castro, F.A.S., Vilas-Boas, J.P., Guimaraes, A.C.S., 2006. Effect of swimming intensity and breathing in front crawl body roll angles for swimmers and triathletes. Brazilian Journal of Biomechanics 7, 85-90.

Cohen, J., 1988. Statistical Power Analysis for the Behavioural Sciences 2nd ed Lawerence Erlbaum Associates, New Jersey.

Counsilman, J.E., 1968. Science of Swimming. Prentice-Hall, Englewood Cliffs, New Jersey.

Field, A., 2005. Discovering Statistics Using SPSS 2nd ed. SAGE Publications, London.

Payton, C.J., Baltzopoulos, V., Bartlett, R.M., 2002. Contributions of rotations of the trunk and upper extremity to hand velocity during front crawl swimming. Journal of Applied Biomechanics 18, 243-256.

Payton, C.J., Bartlett, R.M., Baltzopoulos, V., Coombs, R., 1999. Upper extremity kinematics and body roll during preferred-side breathing and breath-holding front crawl swimming. Journal of Sports Sciences 17, 689-696.

Pendergast, D.R., Di Prampero, P.E., Craig, Jr., A.B., Wilson, D.R., Rennie, D.W., 1977. Quantitative analysis of the front crawl in men and women. Journal of Applied Physiology 43, 475-479.

Psycharakis, S.G., Sanders, R.H., 2008. Shoulder and hip roll changes during 200-m front crawl swimming. Medicine and Science in Sports and Exercise 40, 2129-2136.

Psycharakis, S.G., Sanders, R.H., 2010. Body roll in swimming: a review. Journal of Sports Sciences 28, 229-236.

Psycharakis, S.G., Sanders, R., Mill, F., 2005. A calibration frame for 3D swimming analysis. In: Wang, Q. (Ed.), Proceedings of XVII International Symposium on Biomechanics in Sports. The China Institute of Sports Sciences, Beijing, pp. 901-905.

Psycharakis, S.G., Naemi, R., Connaboy, C., McCabe, C., Sanders, R.H., 2010. Threedimensional analysis of intracycle velocity fluctuations in frontcrawl swimming. Scandinavian Journal of Medicine and Science in Sports 20, 128-135.

Weldon 3rd, E.J., Richardson, A.B., 2001. Upper extremity overuse injuries in swimming. a discussion of swimmer's shoulder. Clinics in Sports Medicine 20 423-438.

Yanai, T., 2001. What causes the body to roll in front-crawl swimming? Journal of Applied Biomechanics 17, 28-42.

Yanai, T., 2003. Stroke frequency in front crawl: its mechanical link to the fluid forces required in non-propulsive directions. Journal of Biomechanics 36, 53-62. 